\title{
OBLIQUE AND CORRELATION ECE IN TCV
}

\author{
T.P. GOODMAN*, V.S. UDINTSEV, E. FABLE, F. FELICI, A. POCHELON, L. \\ PORTE, M. RANCIC, O. SAUTER, CH. SCHLATTER, C. ZUCCA \\ Centre de Recherches en Physique des plasmas, Association \\ EURATOM-Confédération Suisse, Ecole Polytechnique Fédérale de Lausanne (EPFL), \\ CH-1015 Lausanne, Switzerland \\ *E-mail: timothy.goodman@epfl.ch \\ crppwww.epfl.ch
}

\section{P.K. CHATTOPADYAY}

Institute for Plasma Research, Bhat, Gandhinagar-38428, India

The Tokamak à Configuration Variable, TCV, is equipped with a moveable ECE receiving antenna, identical to the 6 second harmonic (X2) ECH/ECCD launchers, which can track the plasma at any position within the vacuum vessel in real time. In contrast to this poloidal plane viewing, the receiver can be rotated to obtain an oblique view. In this configuration, measurements of the asymmetry of the electron distribution function, EDF, are performed during co/counter ECCD sweeps at constant input power. Direct evidence of the driven current is provided by the ratio of radiation temperatures from co and counter views of identical plasmas. The results are simulated by the NOTECTCV radiation-transfer code in which current profile broadening can be included to reproduce the measurements. Studies of core broadband temperature fluctuations, induced by plasma turbulence, are performed with a high-resolution X2 correlation ECE diagnostic. ECE correlation is measured by two frequency-tunable YIG filters that can be placed between $\mathrm{r} / \mathrm{a}=0-0.9$ using both their wide tuning range, and plasma geometry changes from shot to shot. Evidence of broadband $(20-150 \mathrm{kHz})$ fluctuations with peak frequencies ranging from $20 \mathrm{kHz}$ up to $90 \mathrm{KHz}$ at $\mathrm{r} / \mathrm{a}=0.3-0.8$ in Ohmic sawtooth-free discharges was obtained. The amplitude of the temperature fluctuations decreases with increasing density and thus, increasing collisionality, which is in qualitative agreement with predictions from quasi-linear gyrokinetic calculations performed with the gyrokinetic code GS2. The mixing length heat diffusivity calculated from GS2 decreases with increasing collisionality, as does that obtained from a power balance analysis. The real frequency of the broadband turbulence stays positive over the range of collisionalities explored, indicating dominant TEM turbulence.

Keywords: Oblique ECE, Correlation ECE, Fluctuations, Turbulence 


\section{Introduction}

Microinstabilities and the larger self-organized turbulent structures that result from them are thought to lead to the observed anomalous heat transport measured in tokamaks [1]. The break-up or modification of the larger structures can lead to so-called transport barriers. As the anomalously large heat and particle losses are directly responsible for the size and therefore cost of future fusion reactors, understanding and reducing this turbulence is a major goal in magnetic fusion research.

Turbulence can result from magnetic or electro-static fluctuations in the plasma. The former breaks up the nested structure of the field lines, while the latter produces cross-field transport via particle drifts generated by the fluctuation electric field and the background magnetic field, under certain conditions. In order to quantify the transport due to the fluctuating fields, it is necessary to measure the temperature, density and electric potential fluctuations and the phase relationship between them. This is a very challenging task. In the absence of such detailed measurements much can still be inferred through a comparison of trends in the fluctuation measurements and those of gyrokinetic simulations.

One experimental tool set used to investigate the nature of turbulence relies on correlation techniques to seek out the underlying temperature fluctuations in signals dominated by thermal noise. Several measurement setups are possible [2] to achieve independence of the thermal noise but correlation of the thermal fluctuations. All techniques rely on long-time averaging-tozero of the statistically independent noise. An electron cyclotron emission (ECE) diagnostic can be modified to take advantage of these techniques. Such a system is often referred to as correlation ECE or CECE. On TCV the modification is carried out by adding narrow band frequency filters in the radiometer attached to a single-line-of-sight.

In addition to measurements of the fluctuations, another type of modification to a standard ECE diagnostic - inclining the radiometers line-ofsight (antenna) to provide an oblique view of the plasma - may provide a direct measurement of one consequence of enhanced particle transport, as described in the next paragraph. Measurements made with this antenna are referred to as oblique ECE, or ObECE on TCV.

The higher confinement (reduced heat transport) measured in some plasmas can result from modifications of the magnetic shear. On TCV this is accomplished using electron-cyclotron-current-drive (ECCD). The very localized absorption of millimeter waves in the plasma results in a correspondingly narrow driven current channel. If the fast particles that carry 
the current diffuse across the flux surfaces, the driven current channel is smeared out and may be less effective in modifying the shear. ObECE measurements combined with modeling of the electron distribution function (EDF) have the potential to provide both a direct measurement of the driven current direction, and an indication of the width of the current channel.

This paper discusses results from both of these modified ECE systems on the TCV tokamak, CECE and ObECE. Section 2 outlines, briefly, the ECE system, its correlation ECE branch and moveable ObECE antenna. We then present results of CECE measurements of temperature fluctuations and trends in the fluctuation amplitude with collisionality in Sec. 3. A comparison with the gyrokinetic code GS2 is given in Sec. 4. Measurements made with the ObECE of the driven current channel are described in Sec. 5 and modeling of the current channel width with the emission code NOTECTCV is outlined in Sec. 6. Finally, Sec. 7 summarizes the main results and gives some concluding remarks.

\section{ECE setup on TCV}

The basic CECE and ObECE equipment is described in Refs. 3 and 4, respectively. A few changes to the setups described in those references have been made and are outlined here.

The CECE is done by splitting off power going to the 24 channel profile ECE system which comes from any one of the 3 low-field-side (LFS) antennas. The CECE branch has a single $\mathrm{LO}$ at $63 \mathrm{GHz}$ followed by a power splitter and 2 low-noise amplifiers. The signals are subsequently passed through two, independent, center-frequency-tunable $(6-18 \mathrm{GHz})$, narrow-bandwidth $(100-160 \mathrm{MHz})$, YIG filters. This allows selection of the frequency spacing between the filters to ensure that the thermal noise is not correlated (i.e. the filter bandwidths don't overlap) while maintaining a small spatial separation between the measurement regions in the plasma so that the fluctuations remain correlated. After detection and amplification, the video signals are split into three branches each of which are filtered differently and acquired at $\geq 1 \mathrm{MHz}$. The filtered signals are primarily used for low frequency "MHD" studies (low-pass filtered at $40 \mathrm{kHz}$ ), and thermal fluctuation studies (band-pass filtered between $40 \mathrm{kHz}$ and $250 \mathrm{kHz}$ to avoid aliasing). The final branch is low-pass filtered at $600 \mathrm{kHz}$. In this paper we refer to the fluctuation branch. The changes to the system are the filters and the amplification.

ObECE is carried out using a receiver identical to the 6 launching an- 
tennas of the $3 \mathrm{MW}, \mathrm{X} 2, \mathrm{ECH} \& \mathrm{ECCD}$ auxiliary heating system: it is the so-called "7th launcher". The antenna consists of 2 focussing and 2 flat mirrors. The flat mirror closest to the plasma can sweep the plasma view at up to $\sim 100^{\circ} \mathrm{s}^{-1}$ in a plane inclined at any angle to the toroidal plane, about the major radius of the tokamak: the two most useful planes being the poloidal plane (standard ECE view with $\mathbf{k} \perp \mathbf{B}$ ), or the toroidal plane (ObECE). The main changes to the ObECE system are the installation of a $63.5 \mathrm{~mm}$ diameter $\mathrm{HE}_{11}$ corrugated waveguide at the output of launcher 7 , replacing the one-inch diameter, smooth, circular waveguide, and replacement of the fundamental-waveguide circular polarizer. The $\mathrm{HE}_{11}$ waveguide mode couples well to a free-space Gaussian beam mode, which passes through a free-space grating-polarizer / convertor section and couples to fundamental-rectangular waveguide. The polarization is selected by choosing a polarizer angle which best couples the elliptically polarized plasma emission, at a given oblique angle, to the linearly polarized $\mathrm{TE}_{10}$ mode of the rectangular guide. The rectangular section is followed by a $\mathrm{TE}_{10}$ to $\mathrm{TE}_{11}$-circular convertor, an uptaper to overmoded circular waveguide for long distance transmission, then a similar downtaper to fundamental rectangular waveguide at the radiometer. The grating mirror is identical in design to one of the two used to set the X2 gyrotrons' polarizations.

It should be noted that the ObECE antenna is connected to the LFS radiometer and therefore $\mathrm{CECE}$ can be done through this antenna, though this is not discussed in this paper.

\section{Temperature fluctuation measurements}

Plasmas were positioned in front of the LFS lens antenna located on the machine midplane $(z=0)$ so that the view was through the plasma center and the poloidal direction is transverse to the $\mathbf{k}$-vector of the viewing beam. The YIG center frequencies were chosen so that the resonance location was aligned with the expected minimum of the beam power-spot radius $\left(w_{p}=11 \mathrm{~mm}\right)$ and were separated by $0.24 \mathrm{GHz}$. The thermal noise in this case is not correlated. By choosing the minimum beam spot we maximize the observable $k_{p o l}$ and thus $k_{\perp}$ of the turbulence $\left(k_{r}\right.$ is already much larger than $k_{p o l}$ since the relativistic broadening is small at the low temperatures, $\sim 350 \mathrm{eV}$, involved here). The diagnostic is thus sensitive to $k_{\text {turbulence }} \leq 1.3 \mathrm{~cm}^{-1}$ at $\rho_{v o l} \sim 0.55$ (i.e. $\rho_{v o l}$ is the square-root of the enclosed normalized plasma volume). The spatial separation is $\Delta \rho_{v o l} \sim 0.005$.

Broadband fluctuations are observed between $\sim 20-150 \mathrm{kHz}$ in Ohmically heated plasmas, as seen in Fig. 1; which shows the cross-spectral- 


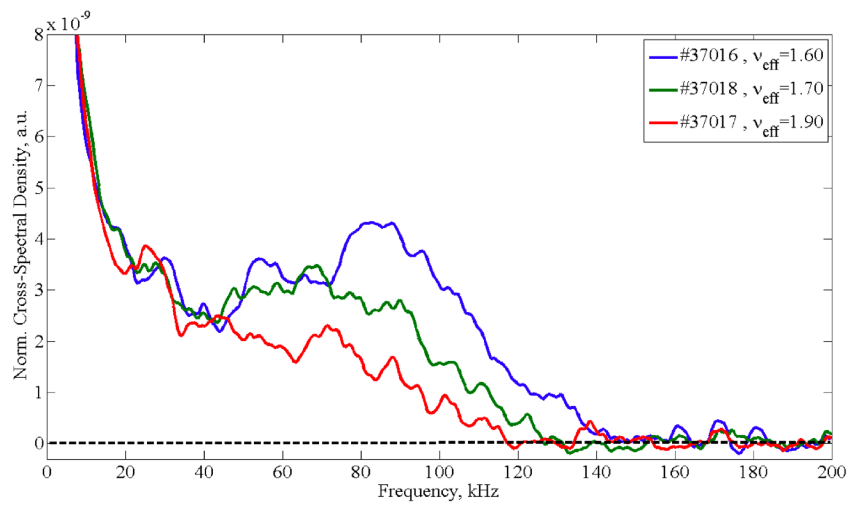

Fig. 1. Broadband fluctuations are visible between $40 \mathrm{kHz}$ and $120 \mathrm{kHz}$. Their amplitude decreases as the collisionality is increased.

density of the signal fluctuations, normalized to their mean values, as a function of frequency. When the density is increased to increase the collisionality, these fluctuations decrease in amplitude. When ECH is added to similar discharges, the fluctuation level increases significantly and the frequency spectrum broadens, as seen in Fig. 2.

In the plasma frame the wave frequency and the wave-number define the phase velocity of the modes: $v_{\text {phase }}=\omega / k$. The plasma $E \times B$ rotation velocity, determined from charge-exchange-spectroscopy measurements of the Carbon impurity in TCV, produces a Doppler-shift in the mode frequency measured by the CECE. DIII-D [5] has reported a broadening of the frequency spectrum due to an increase in the plasma rotation during neutral beam injection. They confirm that the estimated poloidal $\mathbf{k}$-vector component being measured is within the resolution limits of the diagnostic set by the beam spot size, assuming a negligible phase velocity of the mode itself. Similarly, on TCV if the measured peak frequency in the spectrum results from plasma rotation alone (i.e. due to a Doppler-shift of $k_{\text {mode }} v_{\mathbf{E} \times \mathbf{B}}$ ) then $k_{\text {mode }} \sim 2.1 \mathrm{~cm}^{-1} \pm 50 \%$ for shot 37016 of Fig. 1 . The temperature fluctuations that we measure are therefore likely to be strongly attenuated. When the collisionality is increased, the frequency of the peak decreases and the deduced $k_{\text {mode }}$ decreases, so at fixed power-spot radius, the diagnostic sensitivity would increase. Nevertheless, the radiation temperature fluctuation amplitude decreases, as shown in Fig. 3. 


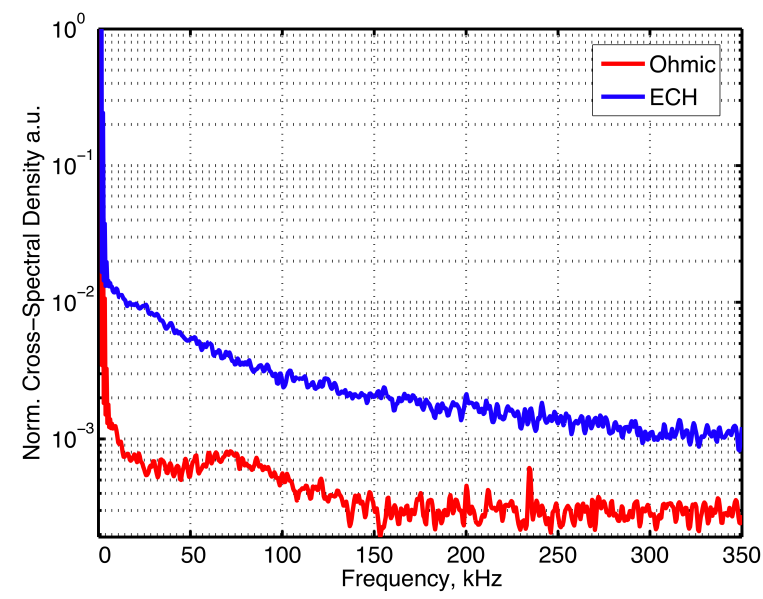

Fig. 2. The broadband fluctuations increase when $500 \mathrm{~kW}$ of ECH is added to the discharge.

\section{Gyrokinetic simulations}

The local gyrokinetic code GS2 [6] is used, along with the quasi-linear model of Ref. [7], to investigate the theoretical trend in fluctuations with increasing collisionality. The calculations are carried out at a radial location of $\rho_{v o l}=0.35$ instead of the experimental $\rho_{v o l}=0.55$ because a larger database of simulated shots already exists at this radius. Other relevant plasma parameters are taken directly from the experimental database (except that only deuterons and electrons are considered, i.e. no impurities). In the simulations $R / L_{n}$ is set to a fixed value of 5 , where $\mathrm{R}$ is the major radius and $L_{n}$ is the density gradient scale length (logarithmic derivative of the density profile at $\left.\rho_{v o l}=0.35\right)$. In the experiments this value is $4.5-6$ $\pm 20 \%$ at $\rho_{\text {vol }}=0.55$. Figure 4 shows the relative density and temperature fluctuation levels (squared) for a mode which is representative of the diagnostic resolution, $k_{p o l}=1.1 \mathrm{~cm}^{-1}$. The average real frequency of this mode remains roughly constant as collisionality is increased, with a sign consistent with TEM turbulence. The mixing-length heat diffusivity calculated from GS2 decreases with increasing collisionality, as does the measured $\chi_{e}$ from a power balance analysis. Together, the trend of these measurements and simulations is consistent with a decrease, by collisional detrapping, of TEM driven losses as collisionality increases. 


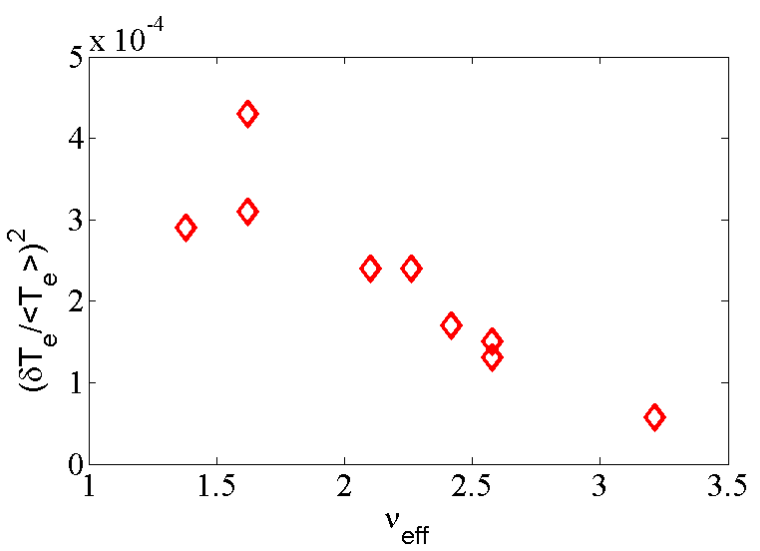

Fig. 3. The measured fluctuation amplitude decreass with increasing collisionality.

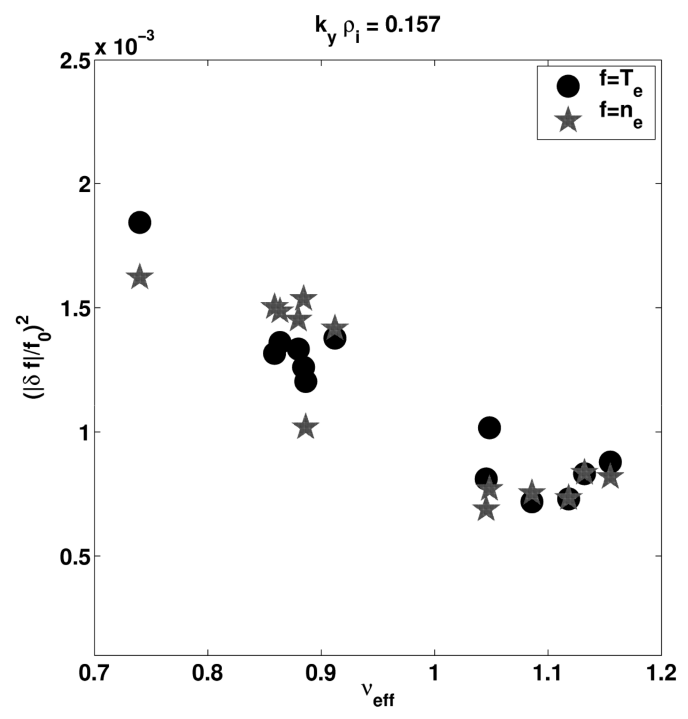

Fig. 4. The GS2 simulated fluctuation amplitudes decrease with increasing collisionality.

\section{Current channel measurements}

The location and sign of the driven current channel from ECCD is put into evidence in a pair of identical discharges where only the viewing direction of the launcher 7 is changed; once looking with the plasma current (co- 
viewing) and once against (counter-viewing). The plasma is heated by 2 clusters of 3 gyrotrons each. Two of the gyrotrons of each cluster heat offaxis. One gyrotron of one cluster drives counter-ECCD on axis and one gyrotron of the other cluster drives co-ECCD in the same location. The power of one cluster is stepped up at the same time as the power from the other cluster is stepped down. This produces a constant input power with a net, centrally driven current which increases from counter- to coECCD in 6 steps. The local modification of the EDF is evidenced by taking the ratio of the co-viewing to counter-viewing radiometer signals, at each frequency, averaged over each of the 6 steps. These ratios from one such pair of shots are shown in Fig. 5. A ratio of 1 indicates an EDF which is symmetric around $v_{\|}=0$. The current is seen to step from the counter to the co direction.

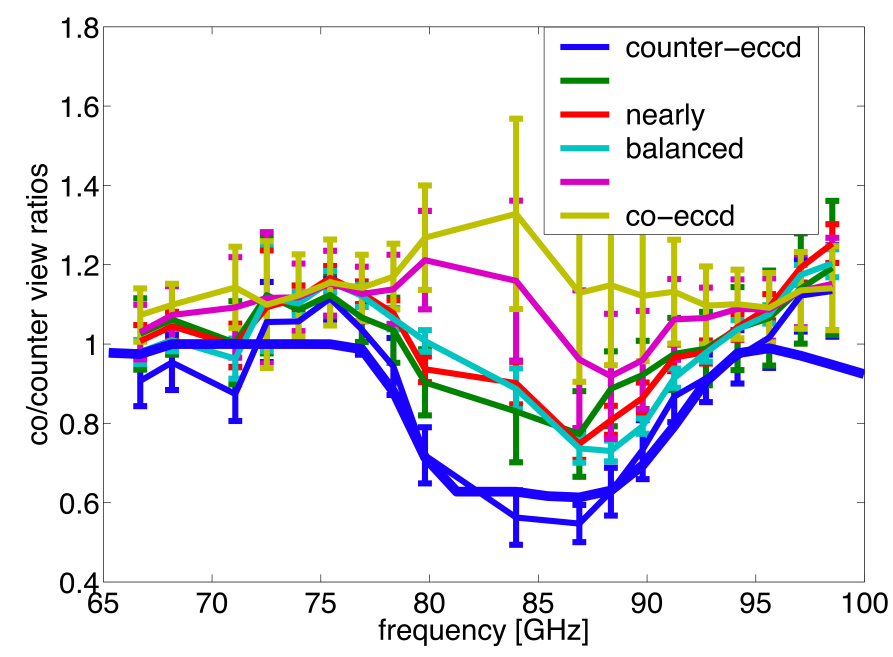

Fig. 5. A central current channel is evident. The solid blue line without errorbars shows the NOTECTCV simluated ratio with current limited within $0<\rho<0.2$. (see text)

\section{Emission simulations}

Note that the radiometer has not been calibrated for this analysis. Only shot-to-shot reproducibility of the plasmas and proper selection of the receiver polarization is required [4]. To provide quantitative information, the ObECE ratios are simulated using the radiation transport code NO- 
TECTCV. The plasma emission is simulated from a sum of up to 20 bi-Maxwellian electron populations drifting, independently, parallel to the magnetic field. Each population can have separate parallel and perpendicular temperatures and can be restricted spatially to a given region in $\rho$. The density of each population is chosen to be proportional to the measured (Thomson Scattering) bulk density profile in that region. Starting with the bulk (essentially non-drifting) population, we keep to a minimum the number of populations needed to reproduce the measured HFS (perpendicular) and LFS (oblique) ECE signals while constraining the drift velocities, densities and regions so as to produce the expected (calculated by the TORAY ray-tracing code) total driven current [8]. The solution is not unique and at present a manual procedure is followed. An example of the match that has been achieved is shown in Fig. 6 for the signals during the counter-ECCD phase of Fig. 5. One bulk, one hot, and one drifting population are required.

To address the question of current diffusion, the above populations were taken as a starting point and the region in which the drifting central current was found was increased while keeping the integrated driven current value constant. Figure 5 shows (thick solid line close to the counter-ECCD measurements) the expected ratio of ObECE signals when the drifting population is extended beyond the maximum $\rho<0.1$ used in Fig. 6 to $\rho<0.2$.

While these results are encouraging, a more self-consistant, less timeconsuming approach is required. Fokker-Planck based ObECE simulations would be best suited to the problem since the wave absorption, particle diffusion and emission can be treated within the same framework.

\section{Conclusions}

Correlation ECE measurements of temperature fluctuations that decrease with collisionality are shown to be consistent with GS2 simulations of fluctuating temperatures and densites. These are accompanied by heat diffusivity measurements which follow the same trend as values determined from a quasi-linear mixing-length model, also based on linear GS2 calculations, which suggests that the anomalous heat loss in these TCV plasmas is the result of TEM generated turbulence: collisional detrapping of particles being the root of the trend with collisionality for this mode.

Oblique ECE measurements of the co- and counter-ECCD current channel are consistent with NOTECTCV simulations of an asymmetric EDF from one drifting bi-Maxwellian population. Current diffusion is required to match the measure co- to counter- viewing ratios of the experiments. A self-consistent simulation approach based on the EDF from the CQL3D 


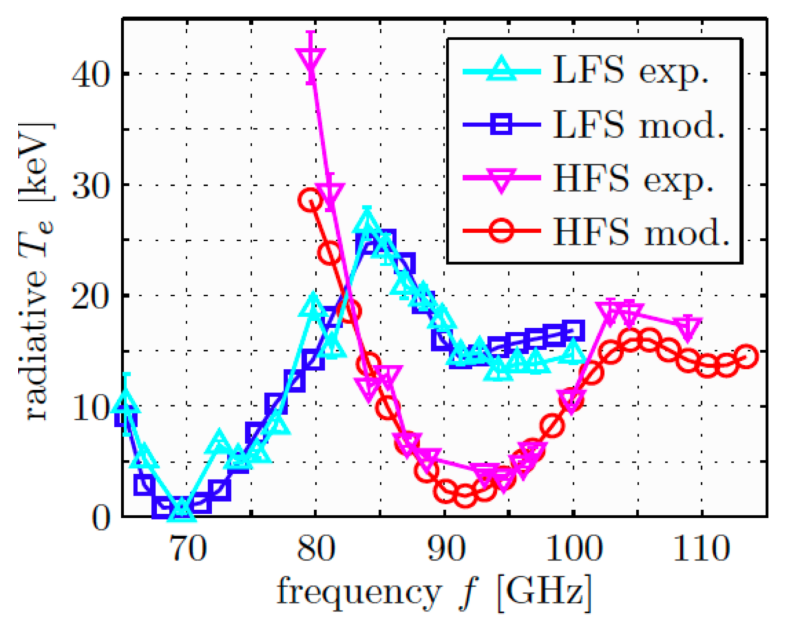

Fig. 6. Simulated and measured HFS and LFS signals during the central counter-ECCD phase of Fig. 5. with the current channel limited to $0<\rho<0.1$. Two populations are required. [taken from Ref. 8]

Fokker-Planck quasi-linear interaction code [9] is being pursued.

\section{Acknowledgments}

The Authors are grateful to W. Dorland and M. Kotschenreuther for having provided the source of the GS2 code. The work was supported in part by the Swiss National Science Foundation.

\section{References}

1. X. Garbet, et al., Nucl. Fusion 50043002 (2010)

2. C. Watts, et al., Proc. of 10th Joint Workshop on Electron Cyclotron Emission and Electron Cyclotron Resonance Heating (Ameland, The Netherlands), (World Scientific), 147 (1997)

3. V.S. Udintsev, et al., Fusion Sci. and Technol. 52, 161-168 (2007)

4. T.P. Goodman, et al., Fusion Sci. and Technol. 53, 196-207 (2008)

5. A. White, et al., Phys. Plasmas, 15, 056116 (2008)

6. M. Kotschenreuther, et al., Comput. Phys. Commun. 88, 128 (1995)

7. E. Fable, et al., Plasma Phys. Control. Fusion 52, 015007 (2010)

8. Ch. Schlatter, "Turbulent Ion Heating in TCV Tokamak Plasmas" EPFL PhD Thesis No. 4479 (2009)

9. R.W. Harvey and M.G. McCoy, Proc. IAEA Technical Meeting on Advances in Simulations and Modeling in Thermonuclear Plasmas (Montreal), (Vienna: IAEA), 498 (1992) 